\title{
Modular, Nondegenerate Polyprotein Scaffolds for Atomic Force Spectroscopy
}

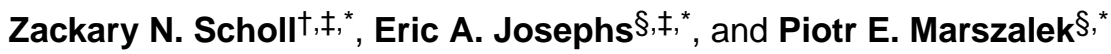 \\ tComputational Biology and Bioinformatics Program, Edmund T. Pratt, Jr. School of Engineering, \\ Duke University, Durham, North Carolina, United States \\ §Department of Mechanical Engineering and Materials Science, Edmund T. Pratt, Jr. School of \\ Engineering, Duke University, Durham, North Carolina, United States
}

\section{INTRODUCTION}

Atomic force spectroscopy (AFS) is a technique in which a cantilever probe of an atomic force microscope (AFM) is used to directly manipulate and pull on individual protein molecules. In an AFS experiment proteins of interest are deposited onto a surface and, after a protein comes into contact with the probe and is mechanically stretched between the surface and the probe, the forces used to unfold the proteins to an unstructured conformation can be directly determined (reviewed in refs 1-7). Such experiments can provide important insights into the energetics of that protein's structure and protein folding or unfolding behavior. ${ }^{8-14}$

"Polyproteins" are polypeptide macromolecules that are composed of tandem identical repeats of protein domains which are each connected by a short linker. ${ }^{1}$ Polyproteins are often used in AFS experiments because they provide several benefits. First, using a larger molecule increases the likelihood of contact between the cantilever probe and the protein. Second, since these incidences of contacts are rare, it is useful to have several proteins on the same molecule so that multiple measurements can be obtained from a single successful pulling event. Third, and most importantly, when these tandem identical repeats are used to flank another protein of interest, the polyprotein can serve (i) as pulling handles that protect the protein of interest from direct contact with the substrate and the AFM tip, and (ii) as a positive control such that the experimenter can be certain that the probe is interacting with a single molecule of interest by the unique "force-extension" signature exhibited by the

*Corresponding Authors. zns@duke.edu, eaj20@duke.edu, pemar@duke.edu.

\#These authors contributed equally (Z.N.S. and E.A.J.).

\section{ASSOCIATED CONTENT}

Supporting Information

The Supporting Information is available free of charge on the ACS Publications website at DOI: 10.1021/acs.biomac.6b00548.

pEMI91 plasmid map, alignment of the shuffled I91 domain sequences, example of sequencing chromatogram, and table and supplementary figure describing force spectroscopy experimental results (PDF). Results of Sanger Sequencing of pEMI91 by sequences $1-7$, T7 promoter, and T7 terminator primers (TXT). Full sequence of pEMI91 (TXT). 
unfolding of the flanking repeats, which can then be differentiated by those produced by the protein of interest. Identical tandem repeats with well-characterized force-extension signatures that have been commonly used as polyproteins include titin I91 domain (formerly known as I27), ${ }^{12,15}$ immunoglobulin G domain GB $1,{ }^{8}$ ubiquitin, ${ }^{16}$ fibronectin, ${ }^{17}$ protein $\mathrm{G},{ }^{18}$ and SNase, ${ }^{19}$ among others.

Though immensely useful, the experimental creation of polyproteins presents a major bottleneck in AFS experiments. To streamline this process, Steward et al. produced a plasmid containing repeating DNA cassettes that code for tandem repeat domains, each of which were separated by unique restriction sites, in a scheme that allows for the DNA coding for the protein of interest to replace individual cassettes with simple cloning techniques. ${ }^{20}$ More recently, Hoffman et al. used Gibson assembly cloning technique to combine DNA cassettes with specially designed linkers to assemble a DNA plasmid coding for a polyprotein, ${ }^{21}$ and Ott et al. ${ }^{22}$ used a Golden Gate-based cloning technique to create tracts of repetitive DNA sequences with controllable lengths. However, in order to perform an AFS experiment successfully, the exact polypeptide sequences of the molecules of interest must be known precisely, while in practice it is very difficult to perform sequencing of the entire DNA sequence coding for the polyprotein to guarantee its fidelity because of the sequence degeneracy of the identical tandem repeat domains. These identical repeats are also subject to uncontrolled expansion or deletion by way of homologous recombination (e.g., as in Supporting Information of ref 13). Nonidentical protein repeats can be used to circumvent this problem, ${ }^{23,24}$ although the force-extension signatures of these polyproteins can be more complex than those from polyproteins derived from a single identical protein, a complication that is nonideal for many force-spectroscopy experiments.

To eliminate this problem, we have improved on earlier polyprotein designs to generate a new plasmid backbone, pEMI91, that resolves these issues by using tandem repeats with "shuffled" codons ${ }^{25,26}$ that can be easily sequenced (Figure 1). The plasmid for the polyprotein was designed to contain nine consecutive cassettes that would each translate to the I91 domain from Homo sapiens TTN gene titin. Each of these cassettes is separated by unique restriction sites for simple replacement of individual I91 cassettes with the DNA coding for a protein of interest, and by virtue of using "unique" I91-coding DNA sequences, a series of primers have been developed that can be used to sequence across the entire polyprotein (Table 1). The polyprotein itself is flanked by a His-tag and a Strep-tag to allow for simplified purification of the expressed protein and possesses two C-terminal cysteine residues to aid in protein display.

Since recent studies on codon shuffling have revealed that modifying codons can affect protein expression, ${ }^{27}$ protein structure, ${ }^{28}$ and folding, ${ }^{29}$ we have verified that our new plasmid has similar properties as earlier examples of titin I91 polyproteins by conducting standard force-spectroscopy experiments to determine that the loading-rate dependence, the distance to transition state, and the unfolding forces. The plasmid coding for this modular, nondegenerate polyprotein scaffold, known as pEMI91, has been deposited into the AddGene repository (\#74888) to be made available for use by researchers. 


\section{MATERIALS AND METHODS}

\section{Protein Purification}

The engineered plasmid was transformed into Escherichia coli BL21(DE3)pLysS cells, and expression was induced using isopropyl $\beta$-D-thiogalactopyranoside. Cell lysate was run through a Strep-tag column (IBA, Goettingen, Germany) and the protein was dialyzed into phosphate-buffered saline (PBS) pH 7.4 and stored in 40\% glycerol and 60\% PBS pH 7.4 at $-20{ }^{\circ} \mathrm{C}$.

\section{Sequencing}

The full plasmid map was sequenced as shown in Figure 1 using the T7 promotor ( $5^{\prime}$ TAATACGACTCACTATAGGG- $3^{\prime}$ ) and the $5^{\prime}-3^{\prime}$ primers in Table 1 , along with the $3^{\prime}-5^{\prime}$ T7 terminator sequencing primer (5'-GGCTTGGTTATGCCGGTACT-3'). Sanger sequencing was performed by Eton Biosciences, Inc.

\section{Atomic Force Microscopy}

Force-spectroscopy measurements were obtained using a custom-built AFM instrument. ${ }^{30}$ Automation routines to control the $\mathrm{AFM}^{31}$ were implemented in LabView (National Instruments, Austin, Texas). Calibration of cantilever spring constants were done in the buffer solution using the energy equipartition theorem. ${ }^{32}$ All measurements were performed in a PBS pH 7.4 solution at room temperature. Force spectroscopy experiments were performed using pulling rates of 50,300,1500, and $3000 \mathrm{~nm} / \mathrm{s}$ using MLCT cantilevers (Bruker, Camarillo, CA) with the spring constant that varied between 16 and $150 \mathrm{pN} / \mathrm{nm}$. In all experiments the purified protein was diluted to $\sim 100 \mu \mathrm{g} / \mathrm{mL}$ in PBS and applied to recently evaporated gold and then incubated for an hour. A worm-like chain (WLC) model ${ }^{33}$ with persistence length of $0.4 \mathrm{~nm}$ was fit to each peak in order to measure contour length increments in the force-extension (FE) data.

\section{Data Analysis}

Between 2000 and 70000 pulls were attempted for each loading rate, until $\sim 20$ high quality curves were obtained with more than three I91 domain unfolding events. The statistics of these attempts and the frequency of the number of domain unfolding events is shown in Supporting Information (Tables S1 and S2). Recordings that had nonspecific events (high force in the beginning of force curve) and recordings that contained events from multiple molecules (contour-length increments much less than prototypical $28 \mathrm{~nm}$ ) were not used for analysis. Typically only $0.03-1.3 \%$ of total pulls resulted in a high quality force-extension curve, while $\sim 63-75 \%$ were empty, and the rest contained nonspecific events or signatures of multiple molecules.

Unfolding forces from usable recordings were binned according to the Freedman-Diaconis rule $^{34}$ and loading rates $\mathrm{d} / \mathrm{d} t$ were calculated using a highly accurate approximation, ${ }^{35}$

$$
\frac{\mathrm{d} F}{\mathrm{~d} t}=v\left[\frac{1}{k_{\mathrm{c}}}+\frac{2 \beta L p(1+\beta F p)}{3+5 \beta F p+8(\beta F p)^{5 / 2}}\right]^{-1}
$$


where $F$ is the force, $v$ is the ramp speed, $k_{\mathrm{c}}$ is the cantilever spring constant, $\beta$ is the reciprocal of thermal energy $\left(\frac{1}{k_{\mathrm{b}} T}\right)$, and $p$ is the persistence length. A persistence length of
$0.365 \mathrm{~nm}$ was used for the fitting.

The histogram of unfolding forces was then combined with the loading rate information to generate the force-dependent unfolding rate data. ${ }^{37,38}$ These data were fit using a model of force-induced unfolding over a barrier, ${ }^{38,39}$ which yielded the unfolding rate, distance to transition state, and the associated errors of estimating these properties.

\section{RESULTS AND DISCUSSION}

A new plasmid sequence containing tandem protein repeats was generated using the pET-15b plasmid sequence as a backbone (Figures 1A and S1, see also Supporting Information for full sequence). To generate nine unique DNA sequences (Supporting Information (SI)), we used amino acid sequence from I91 (UniProtKB Q8WZ42) and reverse translated and then codon shuffled using the Optimizer web service ${ }^{40,41}$ using a random selection of codons weighted by their frequency of use in $E$. coli. The nine tandem I91 domain sequences were checked for sequence disparity using phylogenetic clustering ${ }^{42-44}$ which showed that the minimum proportion of substitutions between any pair of sequences was $8 \%$ (Figures 1B and SI). This high sequence disparity allowed individual primers for each cassette to be designed with high target specificity against the backbone pET-15b plasmid sequence (Table 1) ${ }^{45}$ This polyprotein-coding DNA insert was synthesized by Genscript (Piscataway, NJ) and inserted into the pET-15b backbone.

We tested whether this sequence degeneracy was sufficient to allow us to unambiguously sequence the entire polyprotein-coding DNA using eight designed primers (Materials and Methods) in addition to primers derived from the sequences of the T7 promoter and T7 terminator sites that flank the I91 cassettes. Each primer was able to provide high fidelity sequencing results (Figure S2) up to 800 base pairs and the corresponding sequences matched the designed sequence as intended (red arrows in Figure 1B, see also SI). We verified the protein induction by purifying full-length proteins from the plasmid (details in Materials and Methods) which showed the expected size of $\sim 100 \mathrm{kDa}$ on a SDS-Gel (Figure 2A).

To further verify that our shuffled-sequence polyprotein displayed correct mechanical and folding behavior identical to that of the wild-type 191 polyprotein, we performed standard force-spectroscopy experiments on the expressed polyprotein. The polyprotein comprised of nine tandem I91 domains was pulled at $300 \mathrm{~nm} / \mathrm{s}$ using a cantilever with a spring constant of $16 \mathrm{pN} / \mathrm{nm}$ (more details in Materials and Methods). The schematic of the experiment and representative force-extension curves are shown in Figure 2B. The measured contour-length increment was $28.2 \pm 2.2 \mathrm{~nm}$ (mean and standard deviation) and the unfolding force 202 $\pm 22 \mathrm{pN}$ (Figure 3). These values match well the previously reported values of $28.4 \pm 0.3 \mathrm{~nm}$ and unfolding force of $\approx 200 \mathrm{pN}$. ${ }^{15}$

We also verified the loading-rate dependence of the I9I domains unfolding by performing experiments for an additional three different loading rates (distributions of unfolding forces 
in Figure S3). We followed established procedures for extracting the intrinsic unfolding rate and distance to transition state parameters (see Methods and Materials). The intrinsic unfolding rate determined is $2.1 \pm 1.2 \times 10^{-4} \mathrm{~s}^{-1}$ (mean and standard deviation) and the distance to transition state is $0.35 \pm 0.03 \mathrm{~nm}$. These values compare very well to previous measurements of an unfolding rate of $3.3 \times 10^{-4} \mathrm{~s}^{-1}$ and distance to transition state of $0.25-0.3 \mathrm{~nm} .{ }^{15,46}$

\section{CONCLUSIONS}

A major bottleneck in AFS experiments is the cloning and creation of plasmid sequences for engineered polyproteins that flank a protein of interest, which must contain identical tandem domains to serve as positive control and tethering points. Here we present an improved plasmid backbone with a polyprotein insert containing tandem titin I91 domains with shuffled DNA codons. These domains translate to the prototypical I91 sequence, but allow facile sequencing through their sequence disparity and simple restriction digestion through the incorporation of unique restriction sites. We have verified that this sequence carries the expected properties of previous designs of polyproteins, with the added benefit of full facile sequencing. We expect similar strategies of codon shuffling can be used to generate different polyproteins of interest. The nine I91 polyprotein insert was introduced into a plasmid which is available as a resource to other researchers (AddGene plasmid \#74888).

\section{Supplementary Material}

Refer to Web version on PubMed Central for supplementary material.

\section{Acknowledgments}

This work is supported by the National Science Foundation (NSF) GRFP 1106401 and the Katherine Goodman Stern Fellowship to Z.N.S. and by the NSF MCB-1517245 and MCB-1244297 to P.E.M. P.E.M. acknowledges kind support from the Kimberly-Clark Corporation. E.A.J. is supported by the National Institute of General Medical Sciences of the National Institutes of Health (NIH; F32GM112502).

\section{REFERENCES}

1. Crépin T, Swale C, Monod A, Garzoni F, Chaillet M, Berger I. Curr. Opin. Struct. Biol. 2015; 32:139. [PubMed: 25996897]

2. Ott W, Jobst MA, Schoeler C, Gaub HE, Nash MA. J. Struct. Biol. 2016

3. Popa I, Kosuri P, Alegre-Cebollada J, Garcia-Manyes S, Fernandez JM. Nat. Protoc. 2013; 8:1261. [PubMed: 23744288]

4. Scholl ZN, Li Q, Marszalek PE. Wiley Interdiscip. Rev.: Nanomed. and Nanobiotechnol. 2014; 6:211. [PubMed: 24347439]

5. Tych KM, Hoffmann T, Batchelor M, Hughes ML, Kendrick KE, Walsh DL, Wilson M, Brockwell DJ, Dougan L. Biochem. Soc. Trans. 2015; 43:179. [PubMed: 25849914]

6. Žoldák G, Rief M. Curr. Opin. Struct. Biol. 2013; 23:48. [PubMed: 23279960]

7. Hoffmann T, Dougan L. Chem. Soc. Rev. 2012; 41:4781. [PubMed: 22648310]

8. Cao Y, Li H. Nat. Mater. 2007; 6:109. [PubMed: 17237787]

9. Garcia-Manyes S, Giganti D, Badilla CL, Lezamiz A, Perales-Calvo J, Beedle AE, Fernández JM. J. Biol. Chem. 2016; 291:4226. [PubMed: 26703476]

10. He C, Hu C, Hu X, Hu X, Xiao A, Perkins TT, Li H. Angew. Chem. 2015; 127:10059.

11. Kotamarthi HC, Sharma R, Ainavarapu SRK. Biophys. J. 2013; 104:167a. 
12. Rico F, Gonzalez L, Casuso I, Puig-Vidal M, Scheuring S. Science. 2013; 342:741. [PubMed: 24202172]

13. Scholl ZN, Yang W, Marszalek PE. ACS Nano. 2015; 9:1189. [PubMed: 25639698]

14. Valle-Orero J, Eckels EC, Stirnemann G, Popa I, Berkovich R, Fernandez JM. Biochem. Biophys. Res. Commun. 2015; 460:434. [PubMed: 25796331]

15. Carrion-Vazquez M, Oberhauser AF, Fowler SB, Marszalek PE, Broedel SE, Clarke J, Fernandez JM. Proc. Natl. Acad. Sci. U. S. A. 1999; 96:3694. [PubMed: 10097099]

16. Brujić J, Walther KA, Fernandez JM. Nat. Phys. 2006; 2:282.

17. Li L, Huang HH-L, Badilla CL, Fernandez JM. J. Mol. Biol. 2005; 345:817. [PubMed: 15588828]

18. Cao Y, Balamurali M, Sharma D, Li H. Proc. Natl. Acad. Sci. U. S. A. 2007; 104:15677. [PubMed: 17895384]

19. Wang C-C, Tsong T-Y, Hsu Y-H, Marszalek PE. Biophys. J. 2011; 100:1094. [PubMed: 21320455]

20. Steward A, Toca-Herrera JL, Clarke J. Protein Sci. 2002; 11:2179. [PubMed: 12192073]

21. Hoffmann T, Tych KM, Crosskey T, Schiffrin B, Brockwell DJ, Dougan L. ACS Nano. 2015; 9:8811. [PubMed: 26284289]

22. Ott W, Nicolaus T, Gaub HE, Nash MA. Biomacromolecules. 2016; 17:1330. [PubMed: 26974874]

23. Schlierf M, Rief M. J. Mol. Biol. 2005; 354:497. [PubMed: 16246362]

24. Li H, Oberhauser AF, Fowler SB, Clarke J, Fernandez JM. Proc. Natl. Acad. Sci. U. S. A. 2000; 97:6527. [PubMed: 10823913]

25. Tang NC, Chilkoti A. Nat. Mater. 2016; 15:419. [PubMed: 26726995]

26. Mi L. Biomacromolecules. 2006; 7:2099. [PubMed: 16827576]

27. Gustafsson C, Govindarajan S, Minshull J. Trends Biotechnol. 2004; 22:346. [PubMed: 15245907]

28. Zhou M, Guo J, Cha J, Chae M, Chen S, Barral JM, Sachs MS, Liu Y. Nature. 2013; 495:111. [PubMed: 23417067]

29. Angov E. Biotechnol. J. 2011; 6:650. [PubMed: 21567958]

30. Oberhauser AF, Marszalek PE, Erickson HP, Fernandez JM. Nature. 1998; 393:181. [PubMed: 9603523]

31. Scholl ZN, Marszalek PE. Ultramicroscopy. 2014; 136:7. [PubMed: 24001740]

32. Florin EL, Rief M, Lehmann H, Ludwig M, Dornmair C, Moy VT, Gaub HE. Biosens. Bioelectron. 1995; 10:895.

33. Marko JF, Siggia ED. Macromolecules. 1995; 28:8759.

34. Freedman D, Diaconis P. Probab. Theory Related Fields. 1981; 57:453.

35. Dudko OK, Hummer G, Szabo A. Proc. Natl. Acad. Sci. U. S. A. 2008; 105:15755. [PubMed: 18852468]

36. Dietz H, Rief M. Proc. Natl. Acad. Sci. U. S. A. 2006; 103:1244. [PubMed: 16432239]

37. Evans, E.; Halvorsen, K.; Kinoshita, K.; Wong, WP. Handbook of Single-Molecule Biophysics. Springer; 2009. p. 571

38. Zhang Y, Dudko OK. Proc. Natl. Acad. Sci. U. S. A. 2013; 110:16432. [PubMed: 24062442]

39. Dudko OK, Hummer G, Szabo A. Phys. Rev. Lett. 2006; 96:108101. [PubMed: 16605793]

40. Puigbò P, Guzmán E, Romeu A, Garcia-Vallvé S. Nucleic Acids Res. 2007; 35:W126. [PubMed: 17439967]

41. Puigbò P, Romeu A, Garcia-Vallvé S. Nucleic Acids Res. 2007; 36:D524. [PubMed: 17933767]

42. Goujon M, McWilliam H, Li W, Valentin F, Squizzato S, Paern J, Lopez R. Nucleic Acids Res. 2010; 38:W695. [PubMed: 20439314]

43. McWilliam H, Li W, Uludag M, Squizzato S, Park YM, Buso N, Cowley AP, Lopez R. Nucleic Acids Res. 2013; 41:W597. [PubMed: 23671338]

44. Sievers F, Wilm A, Dineen D, Gibson TJ, Karplus K, Li W, Lopez R, McWilliam H, Remmert M, Söding J. Mol. Syst. Biol. 2011; 7:539. [PubMed: 21988835]

45. Ye J, Coulouris G, Zaretskaya I, Cutcutache I, Rozen S, Madden TL. BMC Bioinf. 2012; 13:134.

46. Rief M, Gautel M, Oesterhelt F, Fernandez JM, Gaub HE. Science. 1997; 276:1109. [PubMed: 9148804] 

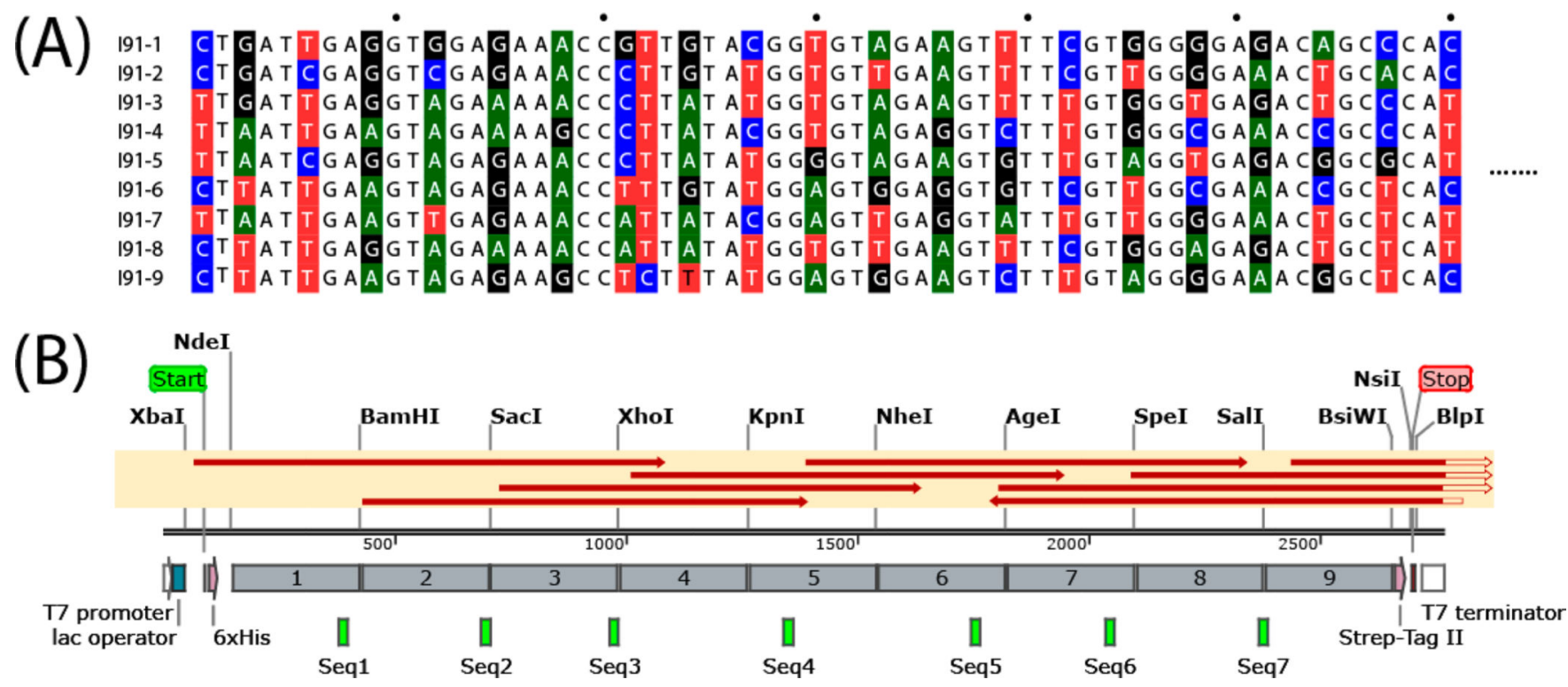

Figure 1.

(A) The DNA sequences from the first $60 \mathrm{bp}$ of each of the nine I91 cassettes colored by their differences to show the shuffling of the codons dispersed throughout the sequence. (B) Schematic of the I91 polyprotein design (gray blocks). The unique restriction sites between each cassette is shown, as well as the placement of a highly specific primer sequence (green blocks labeled Seq1, Seq2, ...). The sequencing coverage by the seven primers plus the T7 terminator and T7 promoter are shown as red arrows above (see also Supporting Information (SI)). The full sequence map is provided at AddGene.org. 


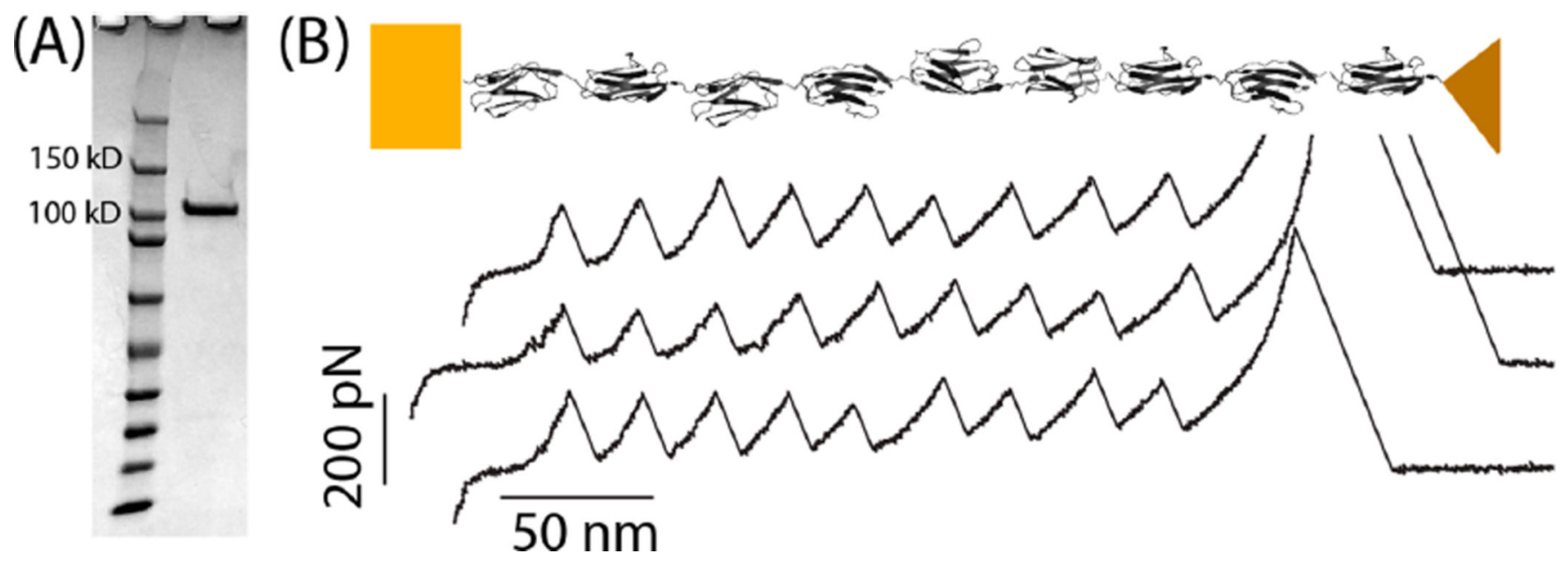

Figure 2.

(A) SDS-gel of the denatured nine I91 domain polyprotein which runs at the expected size $100 \mathrm{kD}$. (B) Schematic of the pulling experiment where the I91 polyprotein is tethered to a gold substrate and pulled from the end by a cantilever, with representative examples of the force-extension signatures that result from unfolding of the nine tandem I91 domains. 

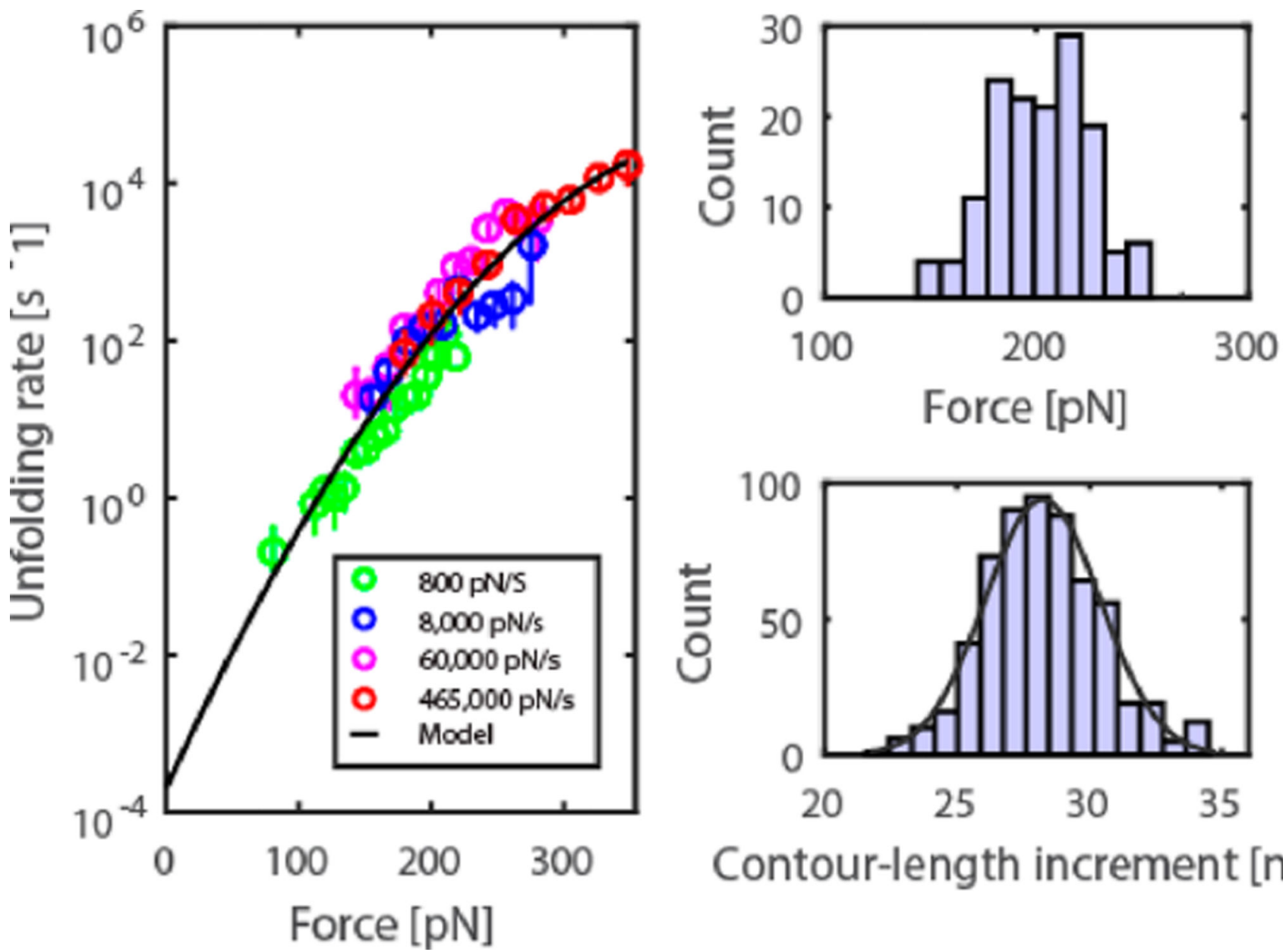

Figure 3.

Left: The unfolding rate versus force data with error bars (dots) fit by a model of the forceinduced unfolding (black line) with an intrinsic unfolding rate of $2.1 \times 10^{-4} \mathrm{~s}^{-1}$ and a distance to transition of $0.35 \mathrm{~nm}$ (see Materials and Methods) Right top: The unfolding force for experiments performed at $300 \mathrm{~nm} / \mathrm{s}$ and spring constant of $16 \mathrm{pN} / \mathrm{nm}$. Right bottom: The contour-length increment of the $\mathrm{I} 91$ domains for all experiments. 


\section{Table 1}

Sequencing Primers for Nondegenerate I91 Polyprotein

\begin{tabular}{cl}
\hline $\begin{array}{c}\text { sequencing primer name } \\
\text { (in Figure 1) }\end{array}$ & DNA sequence \\
\hline Seq1 & $5^{\prime}$-TAACACTAAGAGCGCCGCAA- \\
& $3^{\prime}$ \\
Seq2 & $5^{\prime}$-CAAAGAACTTCGCAGCGAGC- \\
& $3^{\prime}$ \\
Seq3 & $5^{\prime}$-GTAAAAGAACTGCGCTCGCT- \\
& $3^{\prime}$ \\
Seq4 & $5^{\prime}$-ATCTGAACCGGATGTCCACG- \\
& $3^{\prime}$ \\
Seq5 & $5^{\prime}$-GACTGGGGAGGTCAGCTTTC- \\
& $3^{\prime}$ \\
Seq6 & $5^{\prime}$-TGTCCTTTCAGGCCGCTAAT- \\
& $3^{\prime}$ \\
Seq7 & $5^{\prime}$-ATTGCGTAGCGTCGACCTTA- \\
& $3^{\prime}$ \\
\hline
\end{tabular}

